\title{
Approximation of fuzzy numbers by nonlinear Bernstein operators of max-product kind
}

\author{
Lucian Coroianu $^{1}$ Sorin G. Gal ${ }^{2}$ Barnabás Bede ${ }^{3}$ \\ ${ }^{1,2}$ Department of Mathematics and Computer Science, The University of Oradea, \\ Universitatii 1, 410087, Oradea, Romania, E-mails : ${ }^{1}$ lcoroianu@uoradea.ro, ${ }^{2}$ galso@uoradea.ro \\ ${ }^{3}$ Department of Mathematics, The University of Texas-Pan American, \\ 1201 West University, Edinburg, Tx, 78539, USA, E-mail: bedeb@utpa.edu
}

\begin{abstract}
In this paper firstly we extend from $[0,1]$ to an arbitrary compact interval $[a, b]$, the definition of the nonlinear Bernstein operators of max-product kind, $B_{n}^{(M)}(f), n \in \mathbb{N}$, by proving that their order of uniform approximation to $f$ is $\omega_{1}(f, 1 / \sqrt{n})$ and that they preserve the quasi-concavity of $f$. Since $B_{n}^{(M)}(f)$ generates in a simple way a fuzzy number of the same support $[a, b]$ with $f$, it turns out that these results are very suitable in the approximation of the fuzzy numbers. Thus, besides the approximation properties, for sufficiently large $n$, we prove that these nonlinear operators preserve the non-degenerate segment core of the fuzzy number $f$ and, in addition, the segment cores of $B_{n}^{(M)}(f)$, $n \in \mathbb{N}$, approximate the segment core of $f$ with the order $1 / n$.
\end{abstract}

Keywords: fuzzy numbers, nonlinear Bernstein operator of max-product kind

\section{Introduction}

Recently, many papers made investigations on the approximation of fuzzy numbers by trapezoidal or triangular fuzzy members (see [1]-[3], [5]-[6], [12], [16]-[19], [24]-[27], [29]) and by non-linear side functions (see [4], [7], [20], [23], [28]). The main aim of this note is to use the so-called Bernstein operator of max-product kind, firstly introduced (and formally studied) in the book [14], p. 325-326 and completely studied in the papers [9], [8], [13], for approximating fuzzy numbers with continuous membership functions.

The study of max-product type operators started with the papers [10]-[11], where the authors introduced and studied the Shepard nonlinear operator of max-product kind. For a positive continuous function $f:[0,1] \rightarrow \mathbb{R}$, the max-product Bernstein operator was defined in [14], by

$$
B_{n}^{(M)}(f)(x)=\frac{\bigvee_{k=0}^{n} p_{n, k}(x) f(k / n)}{\bigvee_{k=0}^{n} p_{n, k}(x)}, x \in[0,1]
$$

where $p_{n, k}(x)=\left(\begin{array}{l}n \\ k\end{array}\right) x^{k}(1-x)^{n-k}$. Here $\bigvee$ means maximum.
Notice that the max-product Bernstein operator is obtained from the linear Bernstein polynomial written in the form

$$
B_{n}(f)(x)=\frac{\sum_{k=0}^{n} p_{n, k}(x) f(k / n)}{\sum_{k=0}^{n} p_{n, k}(x)},
$$

replacing the "sum" operator by the "maximum" operator.

As it was proved in [8], [9], $B_{n}^{(M)}(f)$ is a continuous nonlinear (more exactly sublinear on the space of positive functions) operator, well-defined for all $x \in \mathbb{R}$, and a piecewise rational function on $\mathbb{R}$. In addition we have $B_{n}^{(M)}(f)(0)=f(0)$ and $B_{n}^{(M)}(f)(1)=f(1)$. Also, in [8] it was proved that $B_{n}^{(M)}(f)$ preserves the monotonicity and the quasiconvexity of $f$ on $[0,1]$ and that the order of uniform approximation by $B_{n}^{(M)}(f)$ is $12 \omega_{1}(f, 1 / \sqrt{n+1})$, where for $g:[0,1] \rightarrow \mathbb{R}$ and $\delta>0$, we take

$$
\begin{gathered}
\omega_{1}(f, \delta)=\sup \{|g(x)-g(y)|: x, y \in[0,1], \\
|x-y| \leq \delta\} .
\end{gathered}
$$

Then, in the paper [13] it was proved that the order of uniform approximation in the whole class $C_{+}([0,1])$ of positive continuous functions on $[0,1]$, cannot be improved, in the sense that there exists a function $f$ in $C_{+}([0,1])$, for which the approximation order by the max-product Bernstein operator is exactly $\omega_{1}(f, 1 / \sqrt{n})$. However, for the class of strictly positive Lipschitz functions, a Jackson type estimate, $\omega_{1}(f, 1 / n)$, was obtained. Finally, in the same paper it was proved that $B_{n}^{(M)}$ preserves the quasi-concavity too.

Now, since the restriction of a continuous fuzzy number to its compact support is a quasi-concave function, naturally it is suggested that $B_{n}^{(M)}$ could be used to approximate a fuzzy number (more correctly the restriction of a fuzzy number to its support). On the other hand, since $B_{n}^{(M)}$ preserves the monotonicity, we can use the operator $B_{n}^{(M)}$ to approximate fuzzy numbers given in the parametric $L U$-form too.

The plan of the paper goes as follows. In Section 2 we define the Bernstein max-product operator on a compact interval $[a, b]$ and prove that the approximation and shape preserving properties one transfer from the case of the $[0,1]$ interval. 
In Section 3, we discuss all the aspects connected to the approximation of fuzzy numbers by these nonlinear operators.

Finally, we present on concrete examples, two graphs which illustrate how the max-product Bernstein operators approximate a fuzzy number and what advantages present with respect to the approximation of fuzzy numbers by the associated linear Bernstein polynomials.

\section{Bernstein max-product operators defined on compact intervals}

From now one, through out this paper, we denote by $C(I)$ and $C_{+}(I)$ respectively, the space of continuous functions defined on an interval $I$ and the space of positive continuous functions defined on $I$ respectively.

For a function $f \in C_{+}([a, b])$, we define the corresponding max-product Bernstein operator by

$B_{n}^{(M)}(f)(x)=\frac{\bigvee_{k=0}^{n} p_{n, k}(x) f\left(a+k \cdot \frac{b-a}{n}\right)}{\bigvee_{k=0}^{n} p_{n, k}(x)}, x \in[a, b]$

where $p_{n, k}(x)=\left(\begin{array}{l}n \\ k\end{array}\right)\left(\frac{x-a}{b-a}\right)^{k} \cdot\left(\frac{b-x}{b-a}\right)^{n-k}$. Since $\sum_{k=0}^{n} p_{n, k}(x)=1$ for all $x \in[a, b]$, it is immediate that $\bigvee_{k=0}^{n} p_{n, k}(x)>0$ for all $x \in[a, b]$ which means that $B_{n}^{(M)}(f)$ is well defined. Also, by simple calculations we get $B_{n}^{(M)}(f)(a)=f(a)$ and $B_{n}^{(M)}(f)(b)=$ $f(b)$. Then, since the maximum of a finite number of continuous functions is a continuous function, we get that for any $f \in C_{+}([a, b]), B_{n}^{(M)}(f) \in C_{+}([a, b])$ too. Actually, if $f:[a, b] \rightarrow \mathbb{R}_{+}$is bounded (not necessarily continuous) then one can easily prove that $B_{n}^{(M)}(f) \in C_{+}([a, b])$.

In this section we will prove that $B_{n}^{(M)}$ : $C_{+}([a, b]) \rightarrow C_{+}([a, b])$ has the same order of uniform approximation as the linear Bernstein operator and that it preserves the quasi-concavity too. First we need the following results and definitions.

Theorem 1 ([8], Theorem 4.1.) If $f:[0,1] \rightarrow \mathbb{R}_{+}$ is continuous then we have the estimate

$$
\left|B_{n}^{(M)}(f)(x)-f(x)\right| \leq 12 \omega_{1}\left(f ; \frac{1}{\sqrt{n+1}}\right),
$$

for all $n \in \mathbb{N}, x \in[0,1]$.

Theorem 2 ([13], Theorem 5.1.) Let us consider the function $f:[0,1] \rightarrow \mathbb{R}_{+}$and let us fix $n \in \mathbb{N}$, $n \geq 1$. Suppose, in addition, that there exists $c \in[0,1]$ such that $f$ is nondecreasing on $[0, c]$ and nonincreasing on $[c, 1]$. Then, there exists $c^{\prime} \in$ $[0,1]$ such that $B_{n}^{(M)}(f)$ is nondecreasing on $\left[0, c^{\prime}\right]$ and nonincreasing on $\left[c^{\prime}, 1\right]$. In addition we have $\left|c-c^{\prime}\right| \leq \frac{1}{n+1}$ and

$$
\left|B_{n}^{(M)}(f)(c)-f(c)\right| \leq \omega_{1}\left(f, \frac{1}{n+1}\right) .
$$

Definition 3 Let $f:[a, b] \rightarrow \mathbb{R}$ be continuous on $[a, b]$. The function $f$ is called:

(i) quasi-convex if

$$
f(\lambda x+(1-\lambda) y) \leq \max \{f(x)), f(y\},
$$

for all $x, y \in[a, b], \lambda \in[0,1]$, (see, e.g. [14], page 4, (iv));

(ii) quasi-concave, if $-f$ is quasi-convex.

Remark 4 By [22], the continuous function $f$ is quasi-convex on $[a, b]$ equivalently means that there exists a point $c \in[a, b]$ such that $f$ is nonincreasing on $[a, c]$ and nondecreasing on $[c, b]$. From the above definition, we easily get that the continuous function $f$ is quasi-concave on $[a, b]$, equivalently means that there exists a point $c \in[a, b]$ such that $f$ is nondecreasing on $[a, c]$ and nonincreasing on $[c, b]$.

We can now present the main results of this section.

Theorem 5 If $a, b \in \mathbb{R}, a<b$ and $f:[a, b] \rightarrow \mathbb{R}_{+}$ is continuous, then we have the estimate

$\left|B_{n}^{(M)}(f)(x)-f(x)\right| \leq 12([b-a]+1) \omega_{1}\left(f ; \frac{1}{\sqrt{n+1}}\right)$,

for all $n \in \mathbb{N}, x \in[a, b]$. Here $\omega_{1}$ denotes the modulus of continuity of $f$ on $[a, b]$.

Proof. Let us consider the function $g:[0,1] \rightarrow$ $\mathbb{R}, g(y)=f(a+(b-a) y)$. It is easy to check that $g(k / n)=f\left(a+k \cdot \frac{b-a}{n}\right)$ for all $k \in\{0,1, \ldots, n\}$. Now, let us choose arbitrary $x \in[a, b]$ and let $y \in[0,1]$ be such that $x=a+(b-a) y$. This implies $y=$ $(x-a) /(b-a)$ and $1-y=(b-x) /(b-a)$. From these equalities and noting the expressions for $g(k / n)$ we obtain $B_{n}^{(M)}(f)(x)=B_{n}^{(M)}(g)(y)$. By Theorem 1 we get

$$
\begin{aligned}
\left|B_{n}^{(M)}(f)(x)-f(x)\right| & =\left|B_{n}^{(M)}(g)(y)-g(y)\right| \\
& \leq 12 \omega_{1}\left(g ; \frac{1}{\sqrt{n+1}}\right) .
\end{aligned}
$$

Since $\omega_{1}\left(g ; \frac{1}{\sqrt{n+1}}\right) \leq \omega_{1}\left(f ; \frac{b-a}{\sqrt{n+1}}\right)$ and from the property $\omega_{1}(f ; \lambda \delta) \leq([\lambda]+1) \omega_{1}(f ; \delta)$ we obtain $\omega_{1}\left(g ; \frac{1}{\sqrt{n+1}}\right) \leq([b-a]+1) \omega_{1}\left(f ; \frac{1}{\sqrt{n+1}}\right)$ and the theorem is proved.

Theorem 6 Let us consider the function $f$ : $[a, b] \rightarrow \mathbb{R}_{+}$and let us fix $n \in \mathbb{N}, n \geq 1$. Suppose in addition that there exists $c \in[a, b]$ such that $f$ is nondecreasing on $[a, c]$ and nonincreasing on $[c, b]$. Then, there exists $c^{\prime} \in[a, b]$ such that $B_{n}^{(M)}(f)$ is nondecreasing on $\left[a, c^{\prime}\right]$ and nonincreasing on $\left[c^{\prime}, b\right]$. In addition we have $\left|c-c^{\prime}\right| \leq \frac{b-a}{n+1}$ and $\left|B_{n}^{(M)}(f)(c)-f(c)\right| \leq([b-a]+1) \omega_{1}\left(f, \frac{1}{n+1}\right)$. 
Proof. We construct the function $g$ as in the previous theorem. Let $c_{1} \in[0,1]$ be such that $g\left(c_{1}\right)=c$. Since $g$ is the composition between $f$ and the linear nondecreasing function $t \rightarrow a+(b-a) t$, we get that $g$ is nondecreasing on $\left[0, c_{1}\right]$ and nonincreasing on $\left[c_{1}, 1\right]$. By Theorem 2 it results that there exists $c_{1}^{\prime} \in[0,1]$ such that $B_{n}^{(M)}(g)$ is nondecreasing on $\left[0, c_{1}^{\prime}\right]$, nonincreasing on $\left[c_{1}^{\prime}, 1\right]$ and in addition we have $\left|B_{n}^{(M)}(g)\left(c_{1}\right)-g\left(c_{1}\right)\right| \leq \omega_{1}\left(g, \frac{1}{n+1}\right)$ and $\left|c_{1}-c_{1}^{\prime}\right| \leq \frac{1}{n+1}$. Let $c^{\prime}=a+(b-a) c_{1}^{\prime}$. If $x_{1}, x_{2} \in\left[a, c^{\prime}\right]$ with $x_{1} \leq x_{2}$ then let $y_{1}, y_{2} \in\left[0, c_{1}^{\prime}\right]$ be such that $x_{1}=a+(b-a) y_{1}$ and $x_{2}=a+(b-a) y_{2}$. Then, it follows that $B_{n}^{(M)}(f)\left(x_{1}\right)=B_{n}^{(M)}(g)\left(y_{1}\right)$ and $B_{n}^{(M)}(f)\left(x_{2}\right)=B_{n}^{(M)}(g)\left(y_{2}\right)$. The monotonicity of $B_{n}^{(M)}(g)$ implies $B_{n}^{(M)}(g)\left(y_{1}\right) \leq B_{n}^{(M)}(g)\left(y_{2}\right)$, that is $B_{n}^{(M)}(f)\left(x_{1}\right) \leq B_{n}^{(M)}(f)\left(x_{2}\right)$. We thus obtain that $B_{n}^{(M)}(f)$ is nondecreasing on $\left[a, c^{\prime}\right]$. Using the same type of reasoning we obtain that $B_{n}^{(M)}(f)$ is nonincreasing on $\left[c^{\prime}, b\right]$. For the rest of the proof, noting that $\left|c_{1}-c_{1}^{\prime}\right| \leq \frac{1}{n+1}$ we get $\left|c-c^{\prime}\right|=$ $\left|(b-a)\left(c_{1}-c_{1}^{\prime}\right)\right| \leq \frac{b-a}{n+1}$. Finally, noting that $\left|B_{n}^{(M)}(g)\left(c_{1}\right)-g\left(c_{1}\right)\right| \leq \omega_{1}\left(g, \frac{1}{n+1}\right)$ and taking into account that $\omega_{1}\left(g, \frac{1}{n+1}\right) \leq([b-a]+1) \omega_{1}\left(f, \frac{1}{n+1}\right)$, we obtain

$$
\begin{aligned}
\left|B_{n}^{(M)}(f)(c)-f(c)\right| & =\left|B_{n}^{(M)}(g)\left(c_{1}\right)-g\left(c_{1}\right)\right| \\
& \leq \omega_{1}\left(g, \frac{1}{n+1}\right) \\
& \leq([b-a]+1) \omega_{1}\left(f, \frac{1}{n+1}\right)
\end{aligned}
$$

and the proof is complete.

Remark 7 From the above theorem and by Remark 4 , it results that if $f:[a, b] \rightarrow \mathbb{R}_{+}$is continuous and quasi-concave then $B_{n}^{(M)}(f)$ is quasi-concave too.

Remark 8 As we have mentioned in the Introduction, for functions in the space $C_{+}([0,1]), B_{n}^{(M)}$ preserves the monotonicity and the quasi-convexity. Reasoning similar as in the proof of Theorem 6, it can be proved that these preservation properties hold in the general case of the space $C_{+}([a, b])$.

\section{Applications in the approximation of fuzzy numbers}

Definition 9 A fuzzy number $u$ is characterized by an upper semicontinuous function $\mu_{u}: \mathbb{R} \rightarrow[0,1]$ with the following properties:

(i) There exists $a, b \in \mathbb{R}, a \leq b$ such that $\mu_{u}(x)=$ 0 outside $[a, b]$.

(ii) There exists $c, d \in \mathbb{R}, c \leq d$ such that:

(ii $\left.i_{1}\right) \mu_{u}$ is nondecreasing on $[a, c]$;

(ii $\left.i_{2}\right) \mu_{u}(x)=1$ for all $x \in[c, d]$;

(ii $i_{2} \mu_{u}$ is nonincreasing on $[d, b]$.

The set $\left\{x \in \mathbb{R}: \mu_{u}(x)=1\right\}$ is called the core of $u$ and it is denoted by $\operatorname{core}(u)$. The closure of the set $\left\{x \in \mathbb{R}: \mu_{u}(x)>0\right\}$ is called the support of $u$ and it is denoted by $\operatorname{supp}(u)$. From the above definition it is immediate that $\operatorname{supp}(u)$ is a bounded interval. If $\mu_{u}$ is continuous and $\operatorname{supp}(u)=[a, b]$, core $(u)=$ $[c, d]$, then we necessarily have $a<c \leq d<b$. For simplicity, from now one we will use the same notation for a fuzzy number and for its membership function.

We need the following auxiliary results.

Lemma 10 Let $a, b \in \mathbb{R}, a<b$. For $n \in \mathbb{N}, k$, $j \in\{0,1, \ldots, n\}$ and $x \in\left(a+j \cdot \frac{b-a}{n+1}, a+(j+1) \cdot \frac{b-a}{n+1}\right)$, let

$$
m_{k, n, j}(x)=\frac{p_{n, k}(x)}{p_{n, j}(x)} .
$$

Then $m_{k, n, j}(x)<1$ for all $j \in\{0,1, \ldots, n\}$ and $k \in$ $\{0,1, \ldots, n\} \backslash\{j\}$.

Proof. Without any loss of generality we may suppose that $a=0$ and $b=1$, because using the same reasoning as in the proof of Theorems 5-6 we easily obtain the conclusion of the lemma in the general case. So, let us fix $x \in(j /(n+1),(j+$ $1) /(n+1))$. According to Lemma 3.2. in [8] we have

$$
\begin{gathered}
m_{0, n, j}(x) \leq m_{1, n, j}(x) \leq \ldots \leq m_{j, n, j}(x) \\
m_{j, n, j}(x) \geq m_{j+1, n, j}(x) \geq \ldots \geq m_{n, n, j}(x)
\end{gathered}
$$

Since $m_{j, n, j}(x)=1$, it suffices to prove that $m_{j+1, n, j}(x)<1$ and $m_{j-1, n, j}(x)<1$. By direct calculations we get

$$
\frac{m_{j, n, j}(x)}{m_{j+1, n, j}(x)}=\frac{j+1}{n-j} \cdot \frac{1-x}{x} .
$$

Since the function $g(y)=(1-y) / y$ is strictly decreasing on the interval $[j /(n+1),(j+1) /(n+1)]$, it results that

$$
\frac{1-x}{x}>\frac{1-(j+1) /(n+1)}{(j+1) /(n+1)}=\frac{n-j}{j+1} .
$$

Clearly, this implies $m_{j, n, j}(x) / m_{j+1, n, j}(x)>1$, that is $m_{j+1, n, j}(x)<1$. By similar reasonings we get that $m_{j-1, n, j}(x)<1$ and the proof is complete.

Lemma 11 If $a, b \in \mathbb{R}, a<b$ and $f:[a, b] \rightarrow \mathbb{R}_{+}$ is bounded then, for all $j \in\{0,1, \ldots, n\}$, we have $B_{n}^{(M)}(f)(a+j(b-a) / n) \geq f(a+j(b-a) / n)$.

Proof. From Lemma 10, since $a+j(b-a) / n \in$ $(a+j(b-a) /(n+1), a+(j+1)(b-a) /(n+1))$ and since $m_{k, n, j}(a+j(b-a) / n)=\frac{p_{n, k}(a+j(b-a) / n)}{p_{n, j}(a+j(b-a) / n)}$ for all $k \in\{0,1, \ldots, n\}$, it follows that $\bigvee_{k=0}^{n} p_{n, k}(a+j(b-a) / n)=p_{n, j}(a+j(b-a) / n)$. Then, we have 


$$
\begin{aligned}
B_{n}^{(M)}(f)(a+j(b-a) / n) \\
\quad=\frac{\bigvee_{k=0}^{n} p_{n, k}(a+j(b-a) / n) f(a+k(b-a) / n)}{p_{n, j}(a+j(b-a) / n)} \\
\geq \frac{p_{n, j}(a+j(b-a) / n) f(a+j(b-a) / n)}{p_{n, j}(a+j(b-a) / n)} \\
=f(a+j(b-a) / n)
\end{aligned}
$$

and the lemma is proved.

Now, suppose that $u$ is a fuzzy number such that $\operatorname{supp}(u)=[a, b]$ and $\operatorname{core}(u)=[c, d]$. For $n \in \mathbb{N}$ we introduce the function $\widetilde{B}_{n}^{(M)}(u)$ : $\mathbb{R} \rightarrow[0,1], \widetilde{B}_{n}^{(M)}(u)(x)=0$ for all $x$ outside $[a, b]$ and $\widetilde{B}_{n}^{(M)}(u)(x)=B_{n}^{(M)}(u)(x)$ for all $x \in[a, b]$. From Theorem 5, it results that the order of uniform approximation of the fuzzy number $u$ by $\widetilde{B}_{n}^{(M)}(u)$ is $\omega_{1}(u, 1 / \sqrt{n})$. Then, since the restriction of $u$ on the interval $[a, b]$ is a function like those considered in Theorem 6 , it results that $\widetilde{B}_{n}^{(M)}(u)$ is a quasiconcave function on $[a, b]$. Moreover, we have the following.

Theorem 12 Let $u$ be a fuzzy number with $\operatorname{supp}(u)=[a, b]$ and $\operatorname{core}(u)=[c, d]$ such that $a \leq c<d \leq b$. Then for sufficiently large $n$, it results that $\widetilde{B}_{n}^{(M)}(u)$ is a fuzzy number such that :

(i) $\operatorname{supp}(u)=\operatorname{supp}\left(\widetilde{B}_{n}^{(M)}(u)\right)$;

(ii) If $\operatorname{core}\left(\widetilde{B}_{n}^{(M)}(u)\right)=\left[c_{n}, d_{n}\right]$, then $\left|c-c_{n}\right| \leq$ $\frac{b-a}{n}$ and $\left|d-d_{n}\right| \leq \frac{b-a}{n}$;

(iii) If, in addition, $u$ is continuous on $[a, b]$, then

$\left|\widetilde{B}_{n}^{(M)}(u)(x)-u(x)\right| \leq 12([b-a]+1) \omega_{1}\left(u ; \frac{1}{\sqrt{n+1}}\right)$,

for all $x \in \mathbb{R}$.

Proof. Let $n \in \mathbb{N}$, such that $\frac{b-a}{n}<d-c$. By Theorem 6 it follows that there exists $c^{\prime} \in[a, b]$ such that $\widetilde{B}_{n}^{(M)}(u)$ is nondecreasing on $\left[a, c^{\prime}\right]$ and nonincreasing on $\left[c^{\prime}, b\right]$. On the other hand, from the definition of $\widetilde{B}_{n}^{(M)}(u)$, it results that $\left\|\widetilde{B}_{n}^{(M)}(u)\right\| \leq\|u\|$ and since $\|u\|=1$, it follows that $\left\|\widetilde{B}_{n}^{(M)}(u)\right\| \leq 1$. (Here $\|\cdot\|$ denotes the uniform norm on $B([a, b])$ the space of bounded functions on $[a, b]$.) Therefore, to prove that $\widetilde{B}_{n}^{(M)}(u)$ is a fuzzy number, it suffices to prove the existence of $\alpha \in[a, b]$ such that $\widetilde{B}_{n}^{(M)}(u)=1$. Let $\alpha=a+j(b-a) / n$ where $j$ is chosen such that $c<\alpha<d$. Such $j$ exists since $\frac{b-a}{n}<d-c$. Since $\alpha \in$ core $(u)$, it results $u(\alpha)=1$. On the other hand, by Lemma 11 it follows that $\widetilde{B}_{n}^{(M)}(u)(\alpha) \geq u(\alpha)$ and clearly this implies that $\widetilde{B}_{n}^{(M)}(u)$ is a fuzzy number. In what follows we prove punctually the rest of the theorem.

(i) As we have mentioned at the beginning of Section $2, B_{n}^{(M)}(u)(a)=u(a)$ and $B_{n}^{(M)}(u)(b)=u(b)$. Noting the definitions of $u$ and $\widetilde{B}_{n}^{(M)}(u)$, it follows that $\widetilde{B}_{n}^{(M)}(u)(x)=0$ outside $[a, b]$. Now, since $u(x)>0$ and $\widetilde{B}_{n}^{(M)}(u)(x)=B_{n}^{(M)}(u)(x)$ for all $x \in(a, b)$, we easily get that $\widetilde{B}_{n}^{(M)}(u)(x)>0$ for all $x \in(a, b)$, which proves (i).

(ii) Since $u$ is nondecreasing on the interval $[a, c]$ and nonincreasing on the interval $[c, b]$, by Theorem 6 it follows that there exists $c^{\prime}(n) \in[a, b]$ such that $\widetilde{B}_{n}^{(M)}(u)$ is nondecreasing on the interval $\left[a, c^{\prime}(n)\right]$ and nondecreasing on the interval $\left[c^{\prime}(n), b\right]$ and, in addition, we have $\left|c-c^{\prime}(n)\right| \leq(b-a) /(n+1)$. On the other hand, $u$ is nondecreasing on the interval $[a, d]$ and nonincreasing on the interval $[d, b]$. It follows that there exists $d^{\prime}(n) \in[a, b]$ such that $\widetilde{B}_{n}^{(M)}(u)$ is nonincreasing on the interval $\left[a, d^{\prime}(n)\right]$ and nondecreasing on the interval $\left[d^{\prime}(n), b\right]$ and, in addition, we have $\left|d-d^{\prime}(n)\right| \leq(b-a) /(n+1)$. For $n \in \mathbb{N}$ satisfying $(b-a) / n<(d-c) / 2$, it results that $c^{\prime}(n)<d^{\prime}(n)$ and this implies that $\widetilde{B}_{n}^{(M)}(u)$ is constant on the interval $\left[c^{\prime}(n), d^{\prime}(n)\right]$, that is $\widetilde{B}_{n}^{(M)}(u)(x)=1$ for all $x \in\left[c^{\prime}(n), d^{\prime}(n)\right]$. This implies that $\left[c^{\prime}(n), d^{\prime}(n)\right] \subseteq \operatorname{core}\left(\widetilde{B}_{n}^{(M)}(u)\right)$, that is $c(n) \leq c^{\prime}(n)$ and $d^{\prime}(n) \leq d(n)$. But we necessarily have $c(n)>a+j_{1}(b-a) / n$ and $d(n)<a+j_{2}(b-a) / n$ where $j_{1}$ and $j_{2}$ are chosen such that $a+j_{1}(b-a) / n<c \leq a+\left(j_{1}+1\right)(b-a) / n$ and $a+\left(j_{2}-1\right)(b-a) / n \leq d<a+j_{2}(b-a) / n$. To prove the first statement, let us observe that (see the proof of Lemma 11) $\bigvee_{k=0}^{n} p_{n, k}\left(a+j_{1}(b-a) / n\right)=p_{n, j_{1}}\left(a+j_{1}(b-a) / n\right)$, which implies that

$$
\begin{aligned}
& \widetilde{B}_{n}^{(M)}(u)\left(a+j_{1}(b-a) / n\right) \\
& =\frac{\bigvee_{k=0}^{n} p_{n, k}\left(a+j_{1}(b-a) / n\right) u(a+k(b-a) / n)}{p_{n, j_{1}}\left(a+j_{1}(b-a) / n\right)} .
\end{aligned}
$$

Let $k_{1} \in\{0,1, \ldots, n\}$ be such that $\bigvee_{k=0}^{n} p_{n, k}(a+$ $\left.j_{1}(b-a) / n\right) u(a+k(b-a) / n)=p_{n, k_{1}}\left(a+j_{1}(b-\right.$ $a) / n) u\left(a+k_{1}(b-a) / n\right)$. If $k_{1}=j_{1}$ then we get $\widetilde{B}_{n}^{(M)}(u)\left(a+j_{1}(b-a) / n\right)=u\left(a+j_{1}(b-a) / n\right)$ and since $a+j_{1}(b-a) / n \notin \operatorname{core}(u)$, we obtain that $\widetilde{B}_{n}^{(M)}(u)\left(a+j_{1}(b-a) / n\right)<1$. If $k_{1} \neq j_{1}$ then By Lemma 10, it results $m_{k_{1}, n, j_{1}}\left(a+j_{1}(b-a) / n\right)<1$ and since $\widetilde{B}_{n}^{(M)}(u)\left(a+j_{1}(b-a) / n\right)=m_{k_{1}, n, j_{1}}(a+$ $\left.j_{1}(b-a) / n\right) \cdot u\left(a+k_{1}(b-a) / n\right)$ we reach to the same conclusion, that is $\widetilde{B}_{n}^{(M)}(u)\left(a+j_{1}(b-a) / n\right)<1$. Now, since $a+j_{1}(b-a) / n, c(n) \in\left[a, d^{\prime}(n)\right]$ (this is immediate since for $(b-a) n<(d-c) / 2$ we have $\left.c<d^{\prime}(n)\right)$ and since $\widetilde{B}_{n}^{(M)}(u)$ is nondecreasing on $\left[a, d^{\prime}(n)\right]$ and noting that $\widetilde{B}_{n}^{(M)}(u)\left(a+j_{1}(b-a) / n\right)<$ $\widetilde{B}_{n}^{(M)}(u)\left(c_{n}\right)$, it follows that we necessarily have $c(n)>a+j_{1}(b-a) / n$. The proof of the statement $d(n)<a+j_{2}(b-a) / n$ is similar and therefore we omit the details. Having in mind the above inequalities, we obtain $c-(b-a) / n<c(n) \leq$ $c^{\prime}(n) \leq c+(b-a) /(n+1)$. This clearly implies that $|c(n)-c| \leq(b-a) / n$. By similar reasonings we obtain that $|d(n)-d| \leq(b-a) / n$ and the proof of statement (ii) is complete.

(iii) The proof is immediate by Theorem 5 , taking 
into account the continuity of $u$.

Remarks (i) If the fuzzy number $u$ is unimodal, that is $c=d$, then $\widetilde{B}_{n}^{(M)}(u)$ is not necessarily a fuzzy number. But if we normalize $\widetilde{B}_{n}^{(M)}(u)$, then we obtain the fuzzy number $\frac{1}{\left\|\widetilde{B}_{n}^{(M)}(u)\right\|} \widetilde{B}_{n}^{(M)}(u)$. (Recall that $\|\cdot\|$ denotes the uniform norm). Since $\widetilde{B}_{n}^{(M)}(u) \rightarrow u$ uniformly, we easily get that

$$
\frac{1}{\left\|\widetilde{B}_{n}^{(M)}(u)\right\|} \widetilde{B}_{n}^{(M)}(u) \rightarrow u
$$

uniformly. Or, for $n \in \mathbb{N}$ we introduce the fuzzy number $u_{n}$ as follows. First, we choose $k(c, n)$ such that

$a+(b-a) \cdot \frac{k(c, n)}{(n+1)} \leq c \leq a+(b-a) \cdot \frac{(k(c, n)+1}{(n+1)}$.

For $x$ outside the interval $(a+(b-a) \cdot(k(c, n)-$ $1) /(n+1), a+(b-a) \cdot(k(c, n)+2) /(n+1))$, we take $u_{n}(x)=u(x)$. For $x \in[a+(b-a) \cdot k(c, n) /(n+$ $1), a+(b-a) \cdot(k(c, n)+1) /(n+1)]$ we take $u_{n}(x)=$ 1. Finally, in the missing intervals we take linear functions so that the continuity of $u_{n}$ is ensured.

In addition, it follows that there exists a constant $C$ independent of $n$, such that

$$
\omega_{1}\left(u_{n} ; \frac{1}{\sqrt{n+1}}\right) \leq C \omega_{1}\left(u ; \frac{1}{\sqrt{n+1}}\right) .
$$

Indeed, it is clear that it suffices to compare the two moduli only on one of the two subintervals (each of them of length $(b-a) /(n+1))$ where $u_{n}(x)$ is a linear function. If $\omega_{1}\left(u_{n} ; 1 / \sqrt{n+1}\right)$ is attained on the left-hand side interval, it easily follows that it is less than

$$
\begin{aligned}
& |u(c)-u[c-2(b-a) /(n+1)]| \\
& \leq[2(b-a)+1] \omega_{1}(u ; 1 /(n+1)) \\
& \leq[2(b-a)+1] \omega_{1}(u ; 1 / \sqrt{n+1}) .
\end{aligned}
$$

If $\omega_{1}\left(u_{n} ; 1 / \sqrt{n+1}\right)$ is attained in an interval where $u_{n}(x)$ is not entirely linear, by decomposing that interval into two consecutive subintervals, such that on one $u_{n}(x)$ is linear and on the other one coincides with $u(x)$ (by construction), by the triangle inequality it easily follows that

$$
\begin{gathered}
\omega_{1}\left(u_{n} ; 1 / \sqrt{n+1}\right) \leq \omega_{1}(u ; 1 / \sqrt{n+1}) \\
+[2(b-a)+1] \omega_{1}(u ; 1 / \sqrt{n+1}) .
\end{gathered}
$$

Now, since $a+(b-a) \cdot k(c, n) / n \in \operatorname{core}\left(u_{n}\right)$, it follows that $u_{n}(a+(b-a) \cdot k(c, n) / n)=1$, which by Lemma 11 implies

$$
\widetilde{B}_{n}^{(M)}\left(u_{n}\right)(a+(b-a) \cdot k(c, n) / n)=1 .
$$

Consequently, we get that $\widetilde{B}_{n}^{(M)}\left(u_{n}\right)$ is a proper fuzzy number. Moreover, since $\lim _{n \rightarrow \infty} \operatorname{core}\left(u_{n}\right)=c$, by Theorem 12, (ii), it results that $\lim _{n \rightarrow \infty}\left(\right.$ core $\left.\widetilde{B}_{n}^{(M)}\left(u_{n}\right)\right)=c$. We prove now that $\widetilde{B}_{n}^{(M)}\left(u_{n}\right) \rightarrow u$, uniformly on $[a, b]$. We have

$$
\begin{aligned}
\left|\widetilde{B}_{n}^{(M)}\left(u_{n}\right)(x)-u(x)\right| \\
\quad \leq\left|\widetilde{B}_{n}^{(M)}\left(u_{n}\right)(x)-u_{n}(x)\right|+\left|u_{n}(x)-u(x)\right| \\
\quad \leq 12([b-a]+1) \omega_{1}\left(u_{n} ; \frac{1}{\sqrt{n+1}}\right) \\
\quad+\left|u_{n}(x)-u(x)\right| \\
\quad \leq 12 C([b-a]+1) \omega_{1}\left(u ; \frac{1}{\sqrt{n+1}}\right) \\
\quad+\left|u_{n}(x)-u(x)\right|
\end{aligned}
$$

Since $\left|u_{n}(x)-u(x)\right| \leq 2([b-a]+1) \omega_{1}\left(u ; \frac{1}{n+1}\right)$, we obtain

$$
\begin{aligned}
& \left|\widetilde{B}_{n}^{(M)}\left(u_{n}\right)(x)-u(x)\right| \\
& \leq 12 C([b-a]+1) \omega_{1}\left(u ; \frac{1}{\sqrt{n+1}}\right) \\
& \quad+2([b-a]+1) \omega_{1}\left(u ; \frac{1}{n+1}\right)
\end{aligned}
$$

and this proves that $\widetilde{B}_{n}^{(M)}\left(u_{n}\right) \rightarrow u$, uniformly on $[a, b]$.

(ii) From Theorem 12 it follows that the maxproduct Bernstein operator, $B_{n}^{(M)}$, is more convenient for approximating fuzzy numbers than the classical linear Bernstein operator, $B_{n}$. While the order of uniform approximation is the same, the max-product Bernstein operator preserves better the shape of the approximated fuzzy number. In fact, it is easy to prove that if the fuzzy number $u$ has a continuous membership function, then as $n$ increases to $\infty$ we have $\left\|B_{n}(u)\right\|<1$. Of course, if we normalize $B_{n}(u)$ then we obtain a fuzzy number (it is known that the linear Bernstein operator preserves the quasi-concavity, see e.g. [21]), but the core of the normalized linear Bernstein operator one reduces to a point which is inconvenient in the case when the core of $u$ is a proper interval.

(iii) For practical considerations it is useful to study the problem of approximating fuzzy numbers that are of Lipschitz-type. For example let us suppose that the fuzzy number $u$ is $\alpha$-Lipschitz on $[a, b]$, of order $\alpha \in(0,1]$, i.e.,

$$
|u(x)-u(y)| \leq M|x-y|^{\alpha}, \forall x, y \in[a, b]
$$

with some absolute constant $M$. By Theorem 12, (iii), we have

$$
\left|\widetilde{B}_{n}^{(M)}(u)(x)-u(x)\right| \leq 12([b-a]+1) M(n+1)^{-\alpha / 2},
$$

Now let $\varepsilon>0$ be arbitrary. Then we have

$$
(12(b-a)+1) M(n+1)^{-\alpha / 2}<\varepsilon,
$$




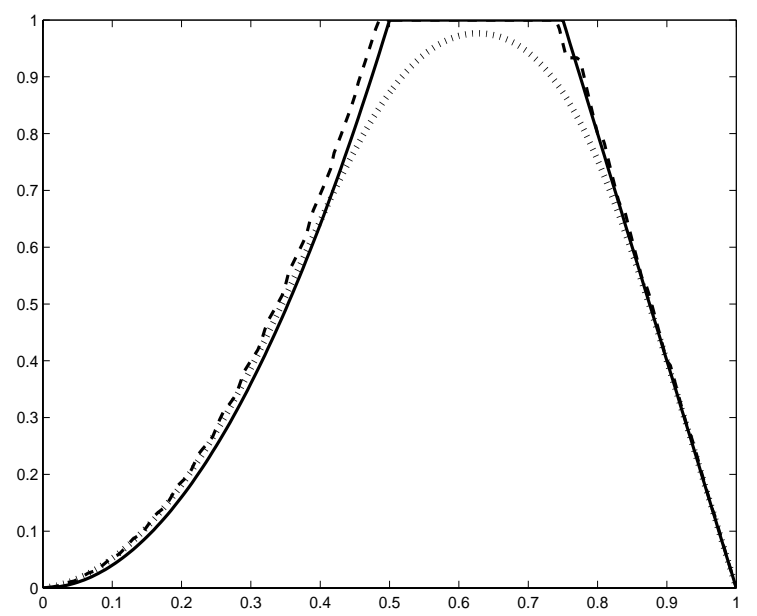

Figure 1: Approximation of a fuzzy number (solid line) by classical (dotted line) and nonlinear Bernstein operators (dashed line) of degree $n=30$.

for any $n \geq n_{0}=\left[\left(\frac{C}{\varepsilon}\right)^{(2 / \alpha)}\right]+1$, with $C=(12(b-$ a) +1$) M$, where [.] stands for the integer part of $x$.

Example 13 We approximate the fuzzy number

$$
u(x)=\left\{\begin{array}{cc}
4 x^{2} & \text { if } 0 \leq x<1 / 2 \\
1 & 1 / 2 \leq x \leq 3 / 4 \\
4-4 x & 3 / 4<x \leq 1 \\
0 & \text { otherwise }
\end{array} .\right.
$$

using both the classical and the nonlinear maxproduct Bernstein operators. In Figures 1 and 2 we can compare the classical and nonlinear maxproduct operators in approximating the above fuzzy number. We can easily see that the classical linear operator marked with dotted line is outperformed by the max-product operator marked with dashed line, this being almost coincident with the target fuzzy number at its core. The theoretical conclusions of the paper are well illustrated by this particular example.

So far, in this section we studied fuzzy numbers given explicitly. It is known that a fuzzy number can be represented in parametric form too. In this case the fuzzy number $u$ is given by a pair of functions $\left(u^{-}, u^{+}\right)$where $u^{-}, u^{+}:[0,1] \rightarrow \mathbb{R}$ satisfy the following requirements:

(i) $u^{-}$is nondecreasing;

(ii) $u^{+}$is nonincreasing;

(iii) $u^{-}(1) \leq u^{+}(1)$.

It is known that for $u=\left(u^{-}, u^{+}\right)$, we have $\operatorname{core}(u)=\left[u^{-}(1), u^{+}(1)\right]$ and $\operatorname{supp}(u)=$ $\left[u^{-}(0), u^{+}(0)\right]$.

If $u=\left(u^{-}, u^{+}\right)$and $v=\left(v^{-}, v^{+}\right)$then (see [15])

$$
\begin{aligned}
d^{2}(u, v) & =\int_{0}^{1}\left(u^{-}(\alpha)-v^{-}(\alpha)\right)^{2} d \alpha \\
& +\int_{0}^{1}\left(u^{+}(\alpha)-v^{+}(\alpha)\right)^{2} d \alpha
\end{aligned}
$$

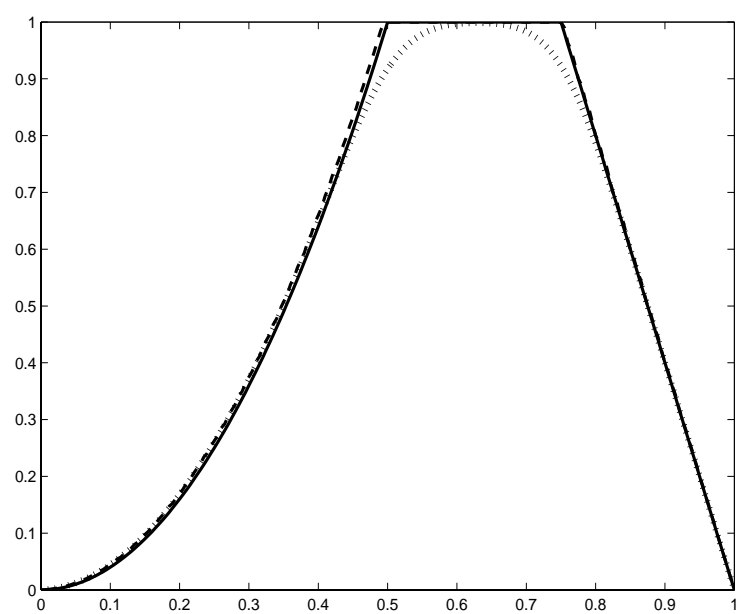

Figure 2: Approximation of a fuzzy number (solid line) by classical (dotted line) and nonlinear Bernstein operators (dashed line) of degree $n=80$.

denotes the Euclidean distance between $u$ and $v$.

For a fuzzy number $u=\left(u^{-}, u^{+}\right)$we attache the Bernstein max-product operators $B_{n}^{(M)}\left(u^{-}\right)$and $B_{n}^{(M)}\left(u^{+}\right)$. Since $B_{n}^{(M)}$ preserves the monotonicity, it is immediate that $B_{n}^{(M)}\left(u^{-}\right)$is nondecreasing and $B_{n}^{(M)}\left(u^{+}\right)$is nonincreasing. In addition we have $B_{n}^{(M)}\left(u^{-}\right)(0)=u^{-}(0), B_{n}^{(M)}\left(u^{-}\right)(1)=$ $u^{-}(1), B_{n}^{(M)}\left(v^{-}\right)(0)=v^{-}(0)$ and $B_{n}^{(M)}\left(v^{-}\right)(1)=$ $v^{-}(1)$. In conclusion we obtain that $\bar{B}_{n}^{(M)}(u)=$ $\left(B_{n}^{(M)}\left(u^{-}\right), B_{n}^{(M)}\left(u^{+}\right)\right)$is a proper fuzzy number which in addition preserves the core and the support of $u$.

We have the following.

Theorem 14 If $u=\left(u^{-}, u^{+}\right)$is a fuzzy number such that $u^{-}$and $u^{+}$are continuous then

$$
\begin{gathered}
d\left(u, \bar{B}_{n}^{(M)}(u)\right) \leq 12 \sqrt{2} \max \left\{\omega_{1}\left(u^{-} ; \frac{1}{\sqrt{n+1}}\right)\right. \\
\left., \omega_{1}\left(u^{+} ; \frac{1}{\sqrt{n+1}}\right)\right\} .
\end{gathered}
$$

Proof. The proof follows from Theorem 1 by applying the mean value theorem for both integrals in the expression of $d\left(u, \bar{B}_{n}^{(M)}(u)\right)$.

Remark 15 Since the linear Bernstein operator preserves the monotonicity, coincides at the end points with the approximated function and has the same order of uniform approximation, it follows that we obtain a similar estimation as in the above theorem if instead of the Bernstein max-product operator we use the linear Bernstein operator.

\section{Conclusions}

In this paper we proved that the Bernstein maxproduct operators might be very useful tools in ap- 
proximating fuzzy numbers. They preserve the support and almost entirely the core of the approximated fuzzy number in the case when the core of the fuzzy number is a proper interval. If the fuzzy number $u$ has a continuous membership function, then the sequence $\widetilde{B}_{n}^{(M)}(u)$ converges to $u$ with respect to the uniform norm and moreover for sufficiently large $n, \widetilde{B}_{n}^{(M)}(u)$ is a proper fuzzy number. For unimodal fuzzy numbers, we presented two methods of approximation by the Bernstein max-product operator. The approximation properties together with the shape preserving properties, indicate that when we approximate a fuzzy number by the Bernstein max-product operator, most of the information associated to a fuzzy number is maintained, which is very important in practice. The complexity of the calculus is at the same level as, for example, in the case of the linear Bernstein operator. For fuzzy numbers given in parametric form with continuous sides, again we obtain the convergence property with respect to the Euclidean distance.

\section{Acknowledgement}

The contribution of the first author was possible with the financial support of the Sectorial Operational Programme for Human Resources Development 2007-2013, co-financed by the European Social Fund, under the project number POSDRU/107/1.5/S/76841 with the title "Modern Doctoral Studies: Internationalization and Interdisciplinarity."

\section{References}

[1] S. Abbasbandy, M.Amirfakhrian, The nearest approximation of a fuzzy quantity in parametric form, Applied Mathematics and Computation, 172 (2006), 624-632.

[2] S. Abbasbandy, M.Amirfakhrian, The nearest trapezoidal form of a generalized left right fuzzy number, International Journal of Approximate Reasoning, 43 (2006), 166-178.

[3] A. I. Ban, Approximation of fuzzy numbers by trapezoidal fuzzy numbers preserving the expected interval, Fuzzy Sets and Systems, 159 (2008), 1327-1344.

[4] A. I. Ban, On the nearest parametric approximation of a fuzzy number-Revisited, Fuzzy Sets and Systems, 160 (2009), 3027-3047.

[5] A. I. Ban, Trapezoidal and triangular approximations of fuzzy numbers-inadvertences and corrections, Fuzzy Sets and Systems 160 (2009), 3048-3058.

[6] A. I. Ban, L. Coroianu, Continuity and Linearity of the trapezoidal approximation preserving the expected interval operator, International Fuzzy Systems Association World Congres, 2024 July 2009, 798-802.
[7] A. I. Ban, L. Coroianu, Metric properties of the neraest extended parametric fuzzy nymbers and applications, International Journal of Approximate Reasoning, 52 (2011) 488-500.

[8] Bede, B., Coroianu, L., Gal, S.G., Approximation and shape preserving properties of the Bernstein operator of max-product kind , Intern. J. Math. and Math. Sci., volume 2009, Article ID 590589, 26 pages, doi:10.1155/2009/590589.

[9] B. Bede, S.G. Gal, Approximation by nonlinear Bernstein and Favard-Szász-Mirakjan operators of max-product kind, Journal of Concrete and Applicable Mathematics, 8 (2010), No. 2, 193-207.

[10] B. Bede, H. Nobuhara, M. Daňková, A. Di Nola, Approximation by pseudo-linear operators, Fuzzy Sets and Systems, 159 (2008), 804820.

[11] B. Bede, H. Nobuhara, J. Fodor, K. Hirota, Max-product Shepard approximation operators, Journal of Advanced Computational Intelligence and Intelligent Informatics, 10 (2006), 494-497.

[12] L. Coroianu, Best Lipschitz constant of the trapezoidal approximation operator preserving the expected interval, Fuzzy Sets and Systems, 165 (2011), 81-97.

[13] L. Coroianu, S. G. Gal, Improved Estimates in Approximation by Bernstein Operators of Max-product kind, Analysis and Applications (to appear).

[14] S.G. Gal, Shape-Preserving Approximation by Real and Complex Polynomials, Birkhäuser, Boston-Basel-Berlin, 2008.

[15] P. Grzegorzewski, Metrics and orders in space of fuzzy numbers, Fuzzy Sets and Systems, 97 (1998), 83-94.

[16] P. Grzegorzewski, Nearest interval approximation of a fuzzy number, Fuzzy Sets and Systems, 130 (2002), 321-330.

[17] P. Grzegorzewski, Trapezoidal approximations of fuzzy numbers preserving the expected interval-Algorithms and properties, Fuzzy Sets and Systems, 47 (2008), 1354-1353.

[18] P. Grzegorzewski, E. Mrówka, Trapezoidal approximations of fuzzy numbers, Fuzzy Sets and Systems, 153 (2005), 115-135.

[19] P. Grzegorzewski, E. Mrówka, Trapezoidal approximations of fuzzy numbers-revisited, Fuzzy Sets and Systems, 158 (2007), 757-768.

[20] E. N. Nasibov, S. Peker, On the nearest parametric approximation of a fuzzy number, Fuzzy Sets and Systems, 159 (2008), 1365-1375.

[21] R. Păltănea, The preservation of the property of quasiconvexity of higher order by Bernstein polynomials, Revue d'Analyse Numér. Théor. Approx., 25 (1996), no. 1-2, 195-201.

[22] T. Popoviciu, Deux remarques sur les function convexes, Bull. Soc. Sci. Acad. Roumaine, 220 
(1938), 45-49.

[23] L. Stefanini, P. Grzegorzewski, Non-linear shaped Approximation of Fuzzy Numbers, International Fuzzy Systems Association World Congres, 20-24 July 2009, 1535-1540.

[24] C-T. Yeh, A note on trapezoidal approximation of fuzzy numbers, Fuzzy Sets and Systems, 158 (2007), 747-754.

[25] C-T. Yeh, On improving trapezoidal and triangular approximations of fuzzy numbers, International Journal of Approximate Reasoning, 48 (2008), 297-313.

[26] C-T. Yeh, Trapezoidal and triangular approximations preserving the expected interval, Fuzzy Sets and Systems, 159 (2008), 13451353.

[27] C-T. Yeh, Weighted trapezoidal and triangular approximations of fuzzy numbers, Fuzzy Sets and Systems, 160 (2009), 3059-3079.

[28] C-T. Yeh, Weighted semi-trapezoidal approximations of fuzzy numbers, Fuzzy Sets and Systems, 165 (2011), 61-80.

[29] W. Zeng, H. Li, Weighted triangular approximation of fuzzy numbers, International Journal of Approximate Reasoning, 46 (2007), 137-150. 